\title{
USGS-OFR--96-557
}

Open-File Report 96-557

\section{Gaining, Losing, and Dry Stream Reaches at Bear Creek Valley, Oak Ridge, Tennessee, March and September 1994}

U.S. GEOLOGICAL SURVEY

Prepared in cooperation with the U.S. DEPARTMENT OF ENERGY
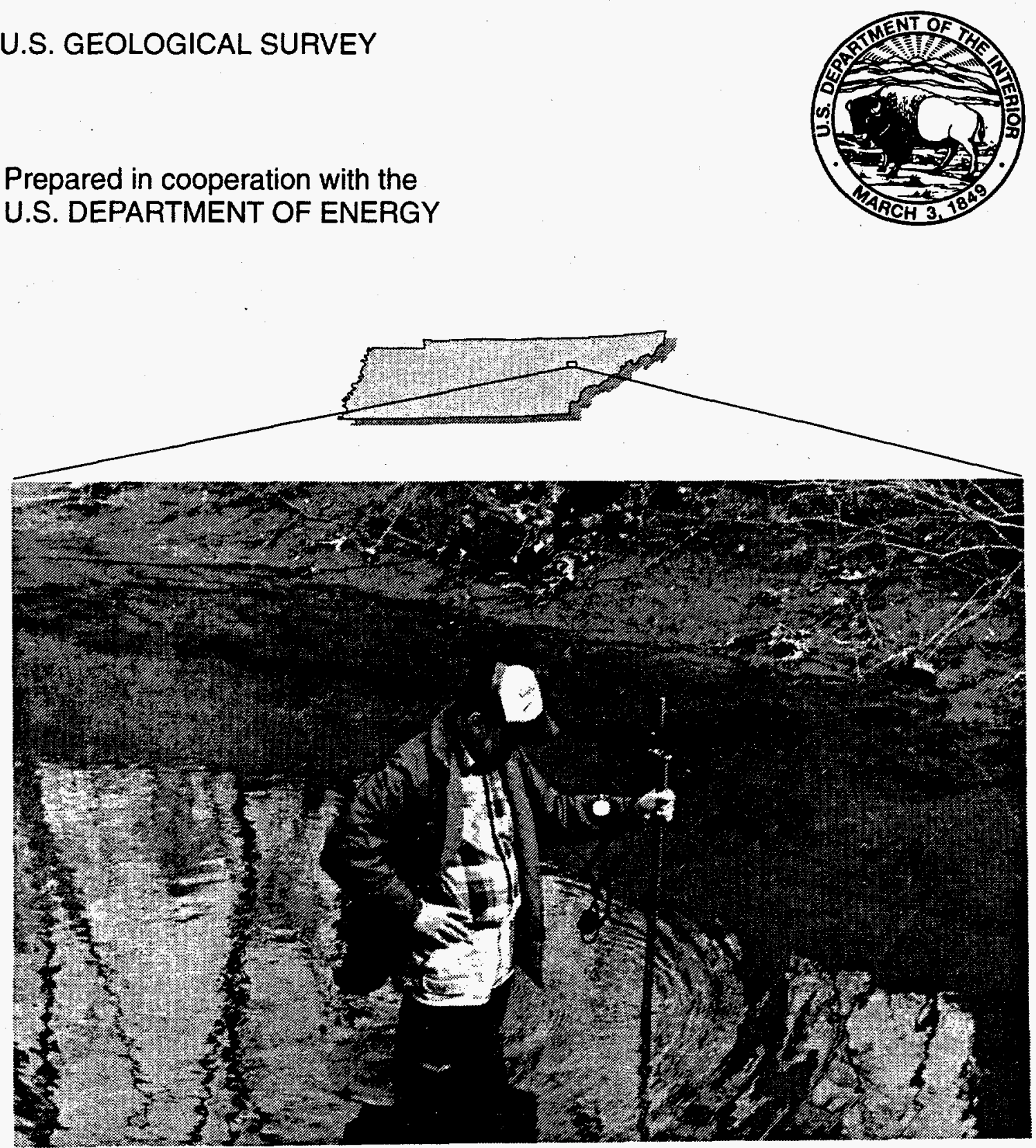

DISTRUTION OF THS DOCUMENT IS URHMTED

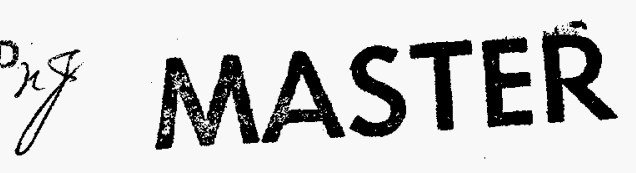


Cover illustration. Photograph by G.E. Hileman. 


\section{DISCLAMMER}

Portions of this document may be illegible in electronic image products. Images are produced from the best available original document. 


\section{GAINING, LOSING, AND DRY STREAM REACHES AT BEAR CREEK VALLEY, OAK RIDGE, TENNESSEE MARCH AND SEPTEMBER 1994}

By John A. Robinson and Reavis L. Mitchell, III

\section{U.S. GEOLOGICAL SURVEY}

Open-File Report 96-557

Prepared in cooperation with the U.S. DEPARTMENT OF ENERGY

\section{DISCLAIMER}

This report was prepared as an account of work sponsored by an agency of the United States Government. Neither the United States Government nor any agency thereof, nor any of their employees, makes any warranty, express or implied, or assumes any legal liability or responsibility for the accuracy, completeness, or usefulness of any information, apparatus, product, or process disclosed, or represents that its use would not infringe privately owned rights. Reference herein to any specific commercial product, process, or service by trade name, trademark, manufacturer, or otherwise does not necessarily constitute or imply its endorsement, recommendation, or favoring by the United States Government or any agency thereof. The views and opinions of authors expressed herein do not necessarily state or reflect those of the United States Government or any agency thereof.

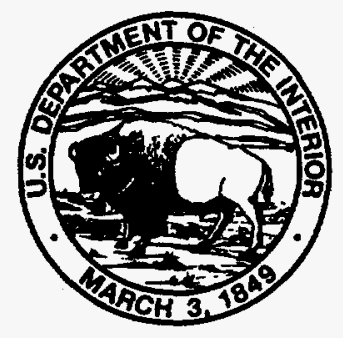




\section{U.S. DEPARTMENT OF THE INTERIOR \\ BRUCE BABBITT, Secretary}

\section{U.S. GEOLOGICAL SURVEY}

Gordon P. Eaton, Director

The use of firm, trade, and brand names in this report is for identification purposes only and does not constitute endorsement by the U.S. Geological Survey

For addtional information write to:

District Chief

U.S. Geological Survey 810 Broadway, Suite 500

Nashville, Tennessee 37203
Copies of this report can be purchased from:

U.S. Geological Survey

Branch of Information Services

Box 25286

Denver, CO 80225-0286 


\section{CONTENTS}

Abstract.

Introduction

Study area

Previous investigation

Gaining, losing, and dry stream reaches

High base flow

Low base flow

Summary

References cited.

\section{P.LATE}

[in pocket]

1. Map showing site locations, discharge measurements, and stream classification during high base flow and low base flow at Bear Creek Valley, Oak Ridge, Tennessee, 1994

\section{FIGURES}

1-3. Maps showing

1. Location of the study area in Bear Creek Valley, Oak Ridge, Tennessee

2. Stream locations and identification numbers for Bear Creek tributaries

3. Location of stream sites in the study area

\section{TABLES}

1. Discharge data during high base flow, March 14 through March 19, and low base flow, September 9 through September 29, 1994, at Bear Creek Valley, Oak Ridge, Tennessee

2. Stream reach classification during high base flow at Bear Creek Valley, Oak Ridge, Tennessee, March 14 through March 19, 1994

3. Stream reach classification during low base flow at Bear Creek Valley, Oak Ridge, Tennessee, September 9 through September 29, 1994

CONVERSION FACTORS AND VERTICAL DATUM

\begin{tabular}{rll}
\hline Multiply & By & To Obtain \\
\hline foot $(\mathrm{ft})$ & 0.3048 & meter \\
acre & 0.4047 & square hectare \\
square mile $\left(\mathrm{mi}^{2}\right)$ & 2.590 & square kilometer \\
mile $(\mathrm{mi})$ & 1.609 & kilometer \\
cubic foot per second $\left(\mathrm{ft}^{3} / \mathrm{s}\right)$ & 0.02832 & cubic meter per second \\
\hline
\end{tabular}

Sea level: In this report "sea level" refers to the National Geodetic Vertical Datum of 1929-a geodetic datum derived from a general adjustment of first-order level nets of the United States and Canada, formally called Sea Level Datum of 1929. 


\title{
Gaining, Losing, and Dry Stream Reaches at Bear Creek Valley, Oak Ridge, Tennessee, March and September 1994
}

\author{
By John A. Robinson and Reavis L. Mitchell, III
}

\section{ABSTRACT}

A study was conducted to delineate stream reaches that were gaining flow, losing flow, or that were dry in the upper reaches of Bear Creek Valley near the Y-12 Plant in Oak Ridge, Tennessee. The study included a review of maps and discharge data from a seepage investigation conducted at Bear Creek Valley; preparation of tables showing site identification and discharge and stream reaches that were gaining flow, losing flow, or that were dry; and preparation of maps showing measurement site locations and discharge measurements, and gaining, losing, and dry stream reaches. This report will aid in developing a better understanding of ground-water and surface-water interactions in the upper reaches of Bear Creek.

\section{INTRODUCTION}

The Oak Ridge Reservation (ORR) is located in East Tennessee in the western part of the Valley and Ridge Physiographic Province. The 58,000-acre ORR is bounded on the northeast, southeast, and southwest by the Clinch River, and on the northwest by Blackoak Ridge (McMaster, 1967). The three major facilities within the ORR are Y-12, a research, development, and production center; $X-10$, the Oak Ridge National Laboratory (ORNL), a research and development center; and K-25, the Gaseous Diffusion Plant (ORGDP), a production center that was closed in 1986.

During 1994 the U.S. Geological Survey (USGS), in cooperation with the U.S. Department of
Energy, conducted a seepage investigation in Bear Creek Valley (Robinson and Johnson, 1996). In 1995, a second study was started to quantify changes in streamflow to delineate gaining, losing, and dry stream reaches in the headwater streams in Bear Creek Valley. Information provided by this report is intended to aid the Y-12 Environmental Restoration Program, Groundwater Operable Units Remedial Investigations Project, to develop a better understanding of groundwater and surface-water interactions in a part of the ORR.

The study involved (1) a review of maps and discharge data from a seepage investigation conducted in Bear Creek Valley; (2) preparation of tables showing site identification and discharge and stream reaches that were gaining flow, losing flow, or that were dry; and (3) preparation of maps showing measurement site locations and discharge measurements, and gaining, losing, and dry stream reaches. All discharge data used in this report were collected during high base flow conditions, March 14 through March 19, 1994, and low base flow conditions, September 9 through September 19, 1994, at Bear Creek Valley, Oak Ridge, Tennessee.

\section{Study Area}

The study area is bounded by Pine Ridge on the northwest, Chestnut Ridge on the southeast, North Tributary 9 (NT9) on the southwest, and the Y-12 Plant on the northeast (fig. 1). Bear Creek Valley southwest of the Y-12 Plant is drained by two streams: Bear Creek, which exits Bear Creek Valley through a water gap in Pine Ridge, and Grassy Creek, which discharges to the Clinch River. The 1,125 acres within the 


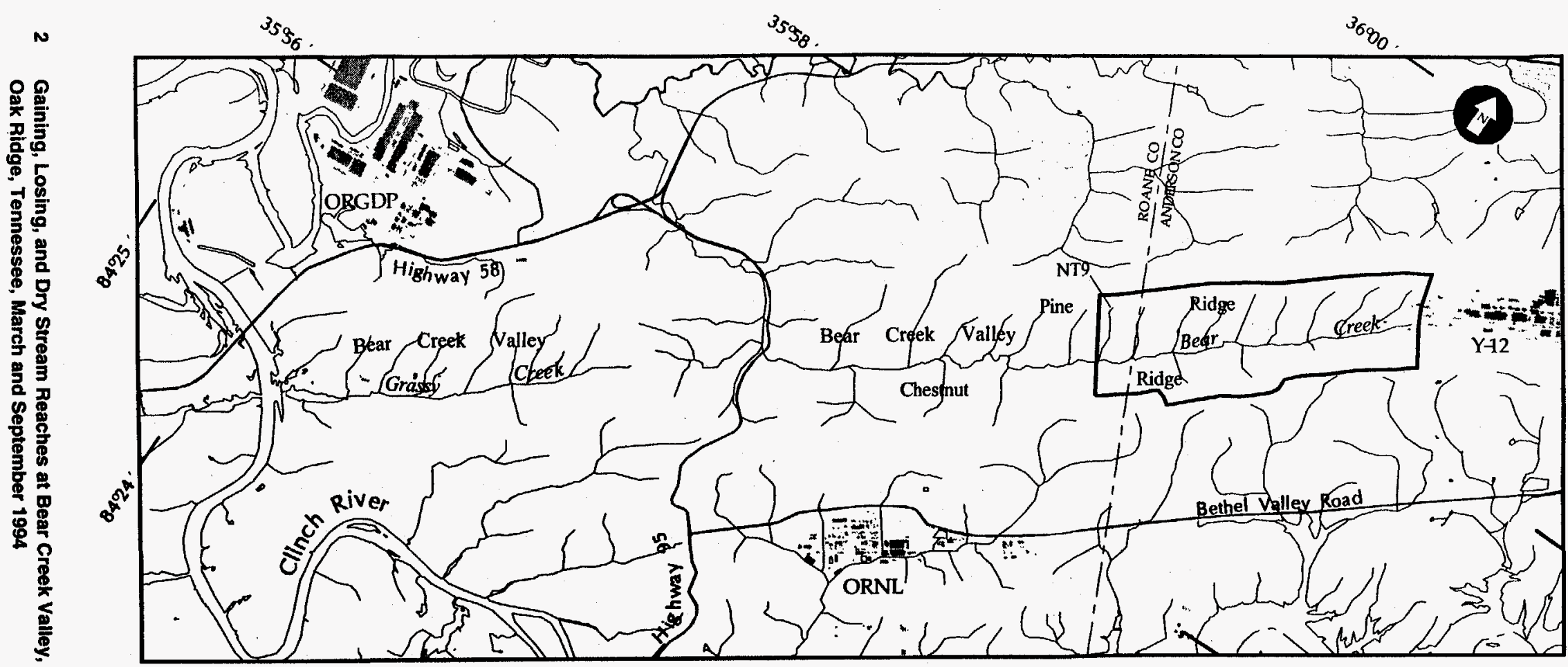

Base from Tennessee Valley Authority S-16A Map of the Oak Ridge, Tennessee Area, December 1987
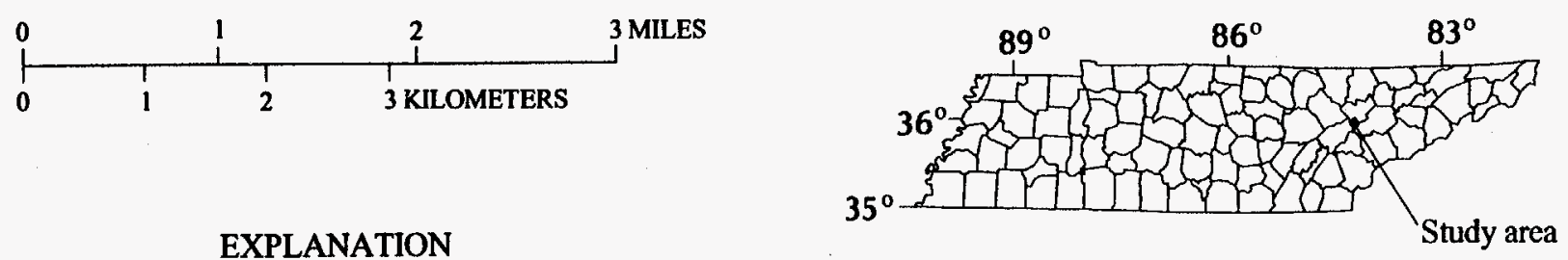

EXPLANATION

- STUDY AREA BOUNDARY

m BUILDINGS

NT9 NORTH TRIBUTARY 9

Figure 1. Location of the study area in Bear Creek Valley, Oak Ridge, Tennessee. 
study area include the uppermost part of Bear Creek and many tributaries north and south of Bear Creek (fig. 2). The main tributaries to the north of Bear Creek include North Tributary 1 (NT1) through NT8. The main tributaries to the south of Bear Creek include South Tributary 1 (ST1) through ST4 and South Spring 1 (SS1) through SS6. In addition to Bear Creek and the main tributaries, many unnamed subtributaries exist throughout the study area.

\section{Previous Investigation}

During 1994 the USGS, in cooperation with the U.S. Department of Energy, conducted a seepage investigation in Bear Creek Valley, in which the Y-12 Plant is located (Robinson and Johnson, 1996). The study involved three phases of activity: (1) a reconnaissance to inventory and map seeps, springs, and stream measurement sites; (2) the measurement of discharge and water-quality characteristics under high base flow conditions; and (3) the measurement of discharge and water-quality characteristics under low base flow conditions. The seepage investigation was conducted on Bear Creek and Grassy Creek. Discharge measurements were made along streams and tributaries in the basins of these two streams, southwest of the Y-12 Plant. In order to gain a better understanding of flow paths in the upper reaches of Bear Creek, discharge measurements from the previous investigation were used in this report to quantify changes in streamflow and to delineate stream reaches which are classified as gaining flow, losing flow, or dry.

\section{GAINING, LOSING, AND DRY STREAM REACHES}

For the purposes of this study, stream reaches were classified as gaining flow (gaining), losing flow (losing), or dry. Changes in streamflow between two adjacent sites on the same stream, plus any flow from contributing tributaries, were used to determine if the flow was gaining or losing along that stream reach. To identify reaches that gain or lose flow, as opposed to apparent differences due to measurement error, the following criteria were used: for streamflow of less than 0.1 cubic foot per second $\left(\mathrm{ft}^{3} / \mathrm{s}\right)$, a change in flow of more than 25 percent of total flow was used to determine if the flow was increasing or decreasing; for streamflow equal to or greater than $0.1 \mathrm{ft}^{3} / \mathrm{s}$, a change in flow of 10 percent was used as the criterion. If two adjacent stream measurement sites had zero flow, these stream reaches were classified as dry. Stream reaches were classified as having no change in flow if the change in streamflow was zero or less than 10 percent for total flow greater than $0.1 \mathrm{ft}^{3} / \mathrm{s}$ or less than 25 percent for total flow between 0.01 and $0.1 \mathrm{ft}^{3} / \mathrm{s}$. If two adjacent stream measurement sites had equal flow rates or the difference in flow rates were in the above specified range, these stream reaches had no change in flow. In many instances, discharge was measured at only one site along a stream reach. These single sites along stream reaches may contribute flow to a stream or tributary. Many of the stream reaches in the study area were unobserved as a result of safety concerns or limited access. Discharge was measured at 229 sites in the study area (fig. 3 ). Site numbers and discharge values are presented in table 1. Changes determined for both high base flow and low base flow conditions, measurement sites, discharge, and stream classification under both conditions are shown on plate 1 at the back of the report.

\section{High Base Flow}

Discharge measurements along upper Bear Creek and its tributaries were collected during high base flow from March 14 through March 19, 1994 (Robinson and Johnson, 1996). During high base flow, discharge measurements for Bear Creek ranged from 0.02 to $2.01 \mathrm{ft}^{3} / \mathrm{s}$; however, site 1490 , on a small tributary to Bear Creek, had a discharge of $0.01 \mathrm{ft}^{3} / \mathrm{s}$ (table 1 , plate 1). No dry stream reaches were observed along Bear Creek during high base flow. Gaining stream reaches occurred near NT2, NT3, NT5, NT7, and NT8; with gains in flow from 0.09 to $0.51 \mathrm{ft}^{3} / \mathrm{s}$. Stream reaches losing flow in the range of 0.13 to $1.34 \mathrm{ft}^{3} / \mathrm{s}$ occurred near NT1, NT2, NT3, NT4, NT5, NT6, and NT7 (table 2).

The discharge measurements along the north tributaries were $0.16 \mathrm{ft}^{3} / \mathrm{s}$ or less with the exception of measurements of 1.10 and $1.16 \mathrm{ft}^{3} / \mathrm{s}$ along NT3 near Bear Creek (table 1, plate 1). All tributaries north of Bear Creek had gaining and losing stream reaches. All north tributaries, except NT3, NT7, and NT8, had dry stream reaches. For most of the tributaries north of Bear Creek, gains in flow were in the range of 0.01 to $0.15 \mathrm{ft}^{3} / \mathrm{s}$ (table 2), except for a section of NT3, which had the largest measured gain in flow of $1.07 \mathrm{ft}^{3} / \mathrm{s}$. Losses in flow for the north tributaries ranged from 0.01 to $0.22 \mathrm{ft}^{3} / \mathrm{s}$ (table 2). 

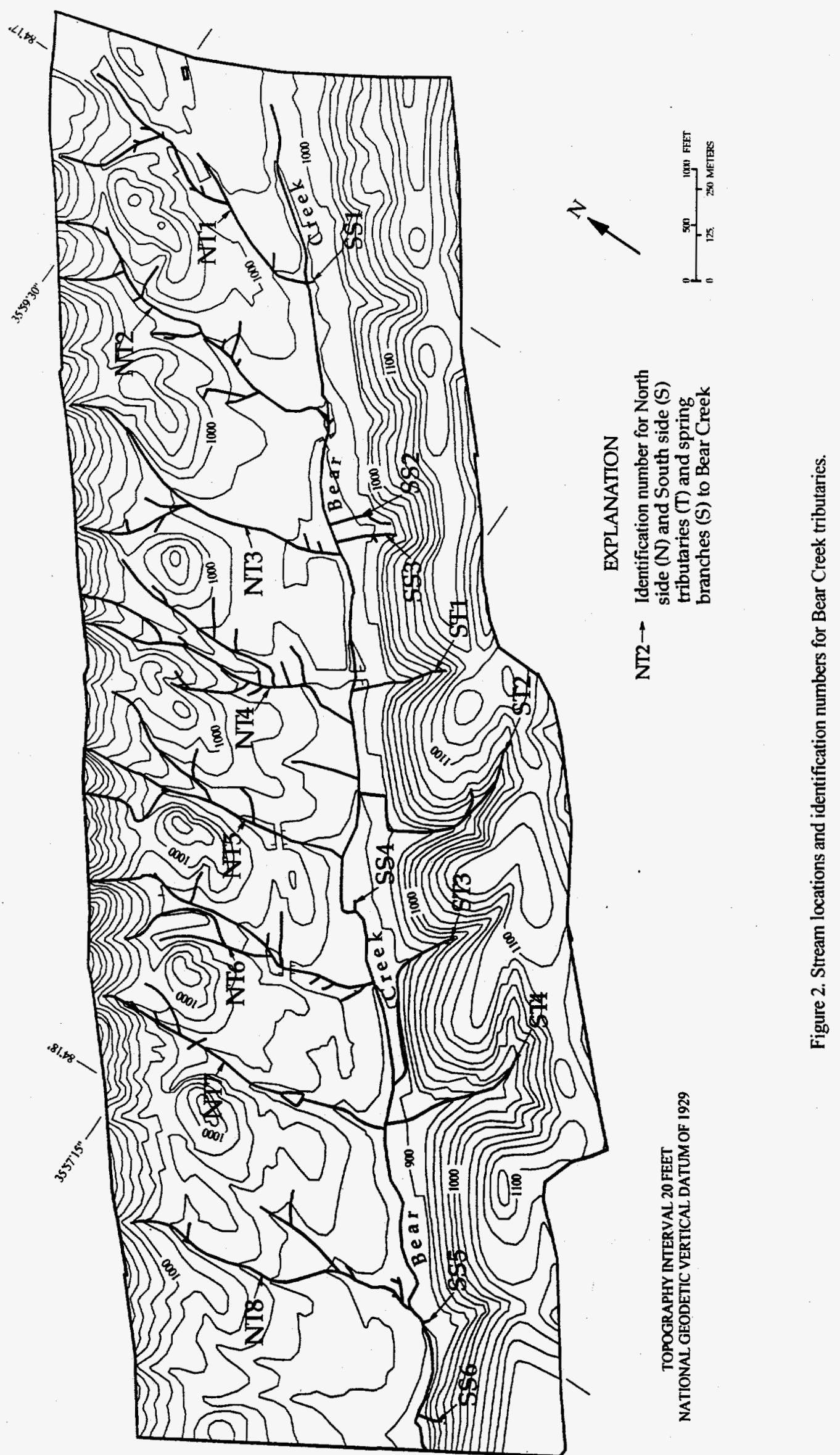

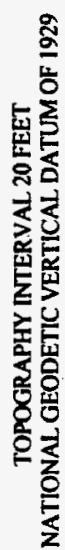

4 Gaining, Losing, and Dry Stream Reaches at Bear Creek Valley, Oak Ridge, Tennessee, March and September 1994 
Discharge measurements along the south tributaries ST1, ST2, and ST4 were mostly zero with the exception of ST3 (table 1, plate 1). Discharge measurements along ST3 ranged from less than 0.02 to $0.44 \mathrm{ft}^{3} / \mathrm{s}$. Stream reaches along ST3 were gaining flow and losing flow in the range of 0.07 to $0.36 \mathrm{ft}^{3} / \mathrm{s}$ (table 2). One dry stream reach was observed along ST2. The south spring tributaries, SS1 through SS6, which may contribute flow to Bear Creek, were mostly single sites with discharge measurements in the range of 0.01 to $0.44 \mathrm{ft}^{3} / \mathrm{s}$ (table 1 ). A section of south spring tributary SS2 had a gain in flow of $0.03 \mathrm{ft}^{3} / \mathrm{s}$ (table 2).

\section{Low Base Flow}

Discharge measurements along upper Bear Creek and its tributaries were collected during low base flow from September 9 through September 29, 1994 (table 1, plate 1). Discharge measurements along Bear Creek ranged from 0 to $0.29 \mathrm{ft}^{3} / \mathrm{s}$. Gaining and losing stream reaches occurred along this section of Bear Creek (table 3). Streamflow gains and losses along Bear Creek were generally in the range of 0.01 to $0.10 \mathrm{ft}^{3} / \mathrm{s}$. The section of Bear Creek near NT4, NT5, and NT6 remained dry. One gaining stream reach along Bear Creek near NT8 had an increase in flow of $0.15 \mathrm{ft}^{3} / \mathrm{s}$.

Most sites on tributaries north of Bear Creek were dry or had flow of $0.01 \mathrm{ft}^{3} / \mathrm{s}$. Only one site on NT8 had a discharge of $0.02 \mathrm{ft}^{3} / \mathrm{s}$ (table 1, plate 1). North tributaries NT1, NT4, and NT5 had gains and losses in flow of $0.01 \mathrm{ft}^{3} / \mathrm{s}$. North tributaries NT6 and NT8 had gains and losses of $0.02 \mathrm{ft}^{3} / \mathrm{s}$.

South tributaries ST1, ST2, and ST4 remained dry during low base flow and ST3 had two discharge measurements of 0.03 and $0.13 \mathrm{ft}^{3} / \mathrm{s}$ (table 1, plate 1). Two gaining reaches occurred along ST3. The south spring tributaries SS1, SS4, and SS6 were dry. South spring tributaries SS2, SS3, and SS5 had discharges of $0.02 \mathrm{ft}^{3} / \mathrm{s}, 0.01 \mathrm{ft}^{3} / \mathrm{s}$, and $0.10 \mathrm{ft}^{3} / \mathrm{s}$, respectively. A stream reach along south spring SS3 was gaining flow at $0.01 \mathrm{ft}^{3} / \mathrm{s}$, and SS5 was a contributing reach to Bear Creek. 

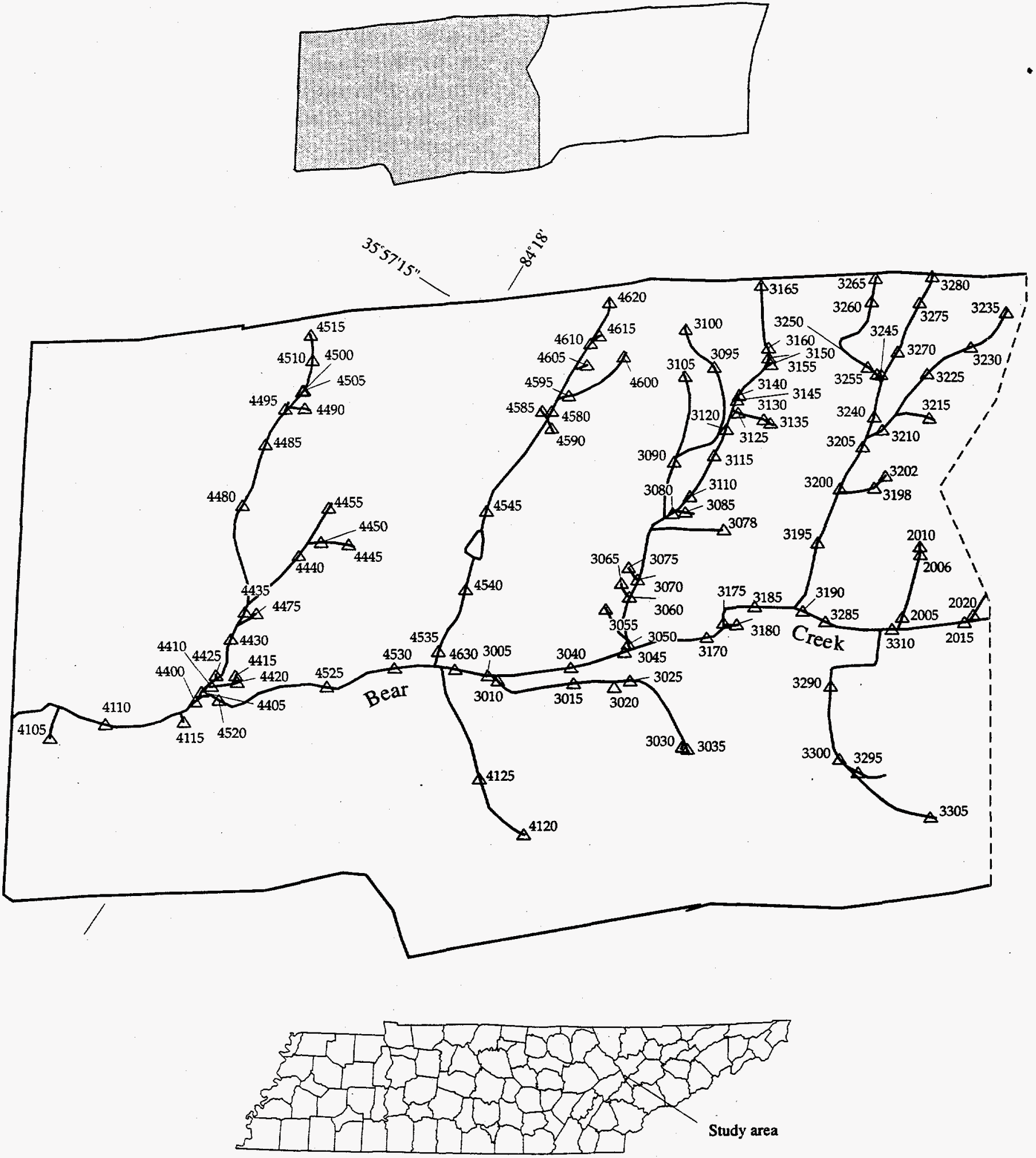

TENNNESSEE

Figure 3. Location of stream sites in the study area.

6 Gaining, Losing, and Dry Stream Reaches at Bear Creek Valley,

Oak Ridge, Tennessee, March and September 1994 


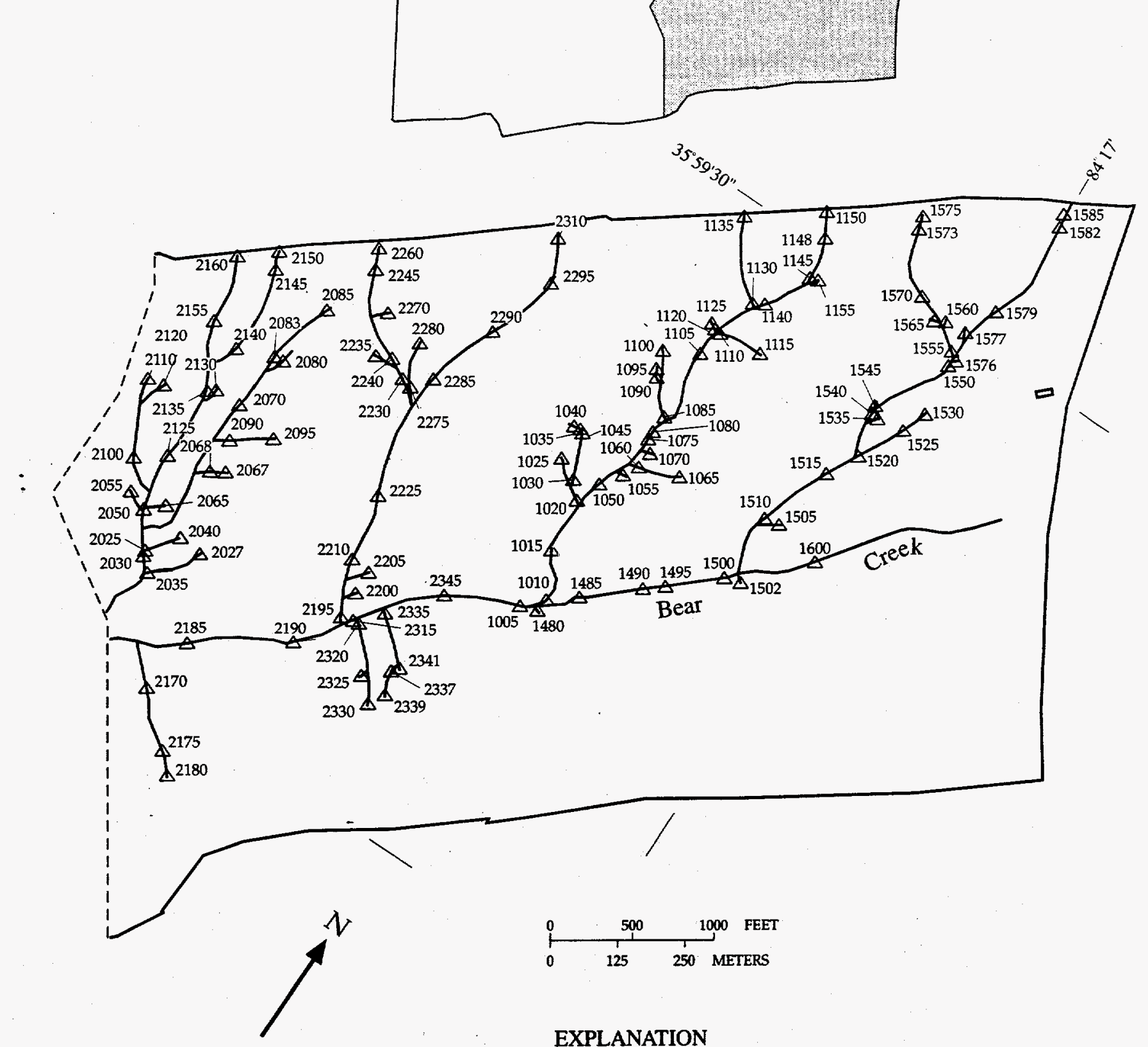

${ }^{2230} \triangle$ SITE LOCATION AND

Figure 3. Location of stream sites in the study area-Continued. 
Table 1. Discharge data during high base flow, March 14 through March 19, 1994, and low base

flow, September 9 through September 29, 1994 at Bear Creek Valley, Oak Ridge, Tennessee

[All discharge values in cubic feet per second; HBF, high base flow; LBF, low base flow]

\begin{tabular}{|c|c|c|c|c|c|c|c|c|}
\hline \multirow{2}{*}{$\begin{array}{c}\text { Site } \\
\text { number }\end{array}$} & \multicolumn{2}{|c|}{ Discharge } & \multirow{2}{*}{$\begin{array}{c}\text { Site } \\
\text { number }\end{array}$} & \multicolumn{2}{|c|}{ Discharge } & \multirow{2}{*}{$\begin{array}{c}\text { Site } \\
\text { number }\end{array}$} & \multicolumn{2}{|c|}{ Discharge } \\
\hline & HBF & LBF & & HBF & LBF & & HBF & LBF \\
\hline \multicolumn{3}{|l|}{ Bear Creek } & \multicolumn{3}{|c|}{ Tributaries to NT1 } & \multicolumn{3}{|c|}{ North Tributary 3 (NT3) } \\
\hline 1490 & 0.01 & 0.00 & 1560 & 0.00 & 0.00 & 2310 & 0.00 & 0.00 \\
\hline 1480 & 0.16 & 0.00 & 1555 & 0.04 & 0.00 & 2295 & 0.04 & 0.00 \\
\hline 1600 & 0.22 & 0.02 & 1545 & 0.00 & 0.00 & 2290 & 0.05 & 0.00 \\
\hline 1500 & 0.30 & 0.01 & 1535 & 0.00 & 0.00 & 2285 & 0.04 & 0.00 \\
\hline 1495 & 0.31 & 0.02 & 1530 & 0.00 & 0.00 & 2225 & 0.03 & 0.00 \\
\hline 3310 & 0.36 & 0.00 & 1525 & 0.01 & 0.00 & 2210 & 1.10 & 0.00 \\
\hline 3040 & 0.39 & 0.00 & 1520 & 0.00 & 0.00 & 2195 & 1.16 & 0.00 \\
\hline 3285 & 0.40 & 0.00 & 1505 & 0.01 & 0.00 & \multirow{2}{*}{\multicolumn{3}{|c|}{ Tributaries to NT3 }} \\
\hline 3190 & 0.14 & 0.00 & & & & & & \\
\hline 2345 & 0.44 & 0.02 & \multicolumn{3}{|c|}{ North Tributary 2 (NT2) } & 2280 & 0.01 & 0.00 \\
\hline 1485 & 0.44 & 0.04 & 1155 & 0.02 & 0.00 & 2275 & 0.01 & 0.00 \\
\hline 3185 & 0.50 & 0.00 & 1140 & 0.03 & 0.00 & 2270 & 0.01 & 0.00 \\
\hline 1005 & 0.57 & 0.02 & 1105 & 0.16 & 0.00 & 2260 & 0.00 & 0.00 \\
\hline 2015 & 0.64 & 0.00 & 1085 & 0.06 & 0.00 & 2245 & 0.02 & 0.00 \\
\hline 3170 & 0.64 & 0.00 & 1080 & 0.00 & 0.00 & 2240 & 0.02 & 0.00 \\
\hline 3045 & 0.69 & 0.00 & 1075 & 0.15 & 0.00 & 2235 & 0.01 & 0.00 \\
\hline 2185 & 0.70 & 0.00 & 1070 & 0.00 & 0.00 & 2230 & 0.03 & 0.00 \\
\hline 3005 & 0.76 & 0.07 & 1055 & 0.00 & 0.00 & 2205 & 0.00 & 0.00 \\
\hline 4630 & 0.79 & 0.07 & 1050 & 0.13 & 0.00 & 2200 & 0.00 & 0.00 \\
\hline 4525 & 0.80 & 0.00 & 1015 & 0.15 & 0.00 & & & \\
\hline 4520 & 0.89 & 0.00 & 1010 & 0.16 & 0.00 & \multicolumn{3}{|c|}{ North Tributary 4 (NT4) } \\
\hline 2190 & 0.90 & 0.04 & & & & 2135 & 0.07 & 0.00 \\
\hline 4530 & 0.93 & 0.08 & \multicolumn{3}{|c|}{ Tributaries to NT2 } & 2125 & 0.04 & 0.00 \\
\hline 4400 & 1.06 & 0.04 & 1150 & 0.01 & 0.00 & 2050 & 0.00 & 0.00 \\
\hline 2315 & 1.08 & 0.00 & 1148 & 0.02 & 0.00 & 2030 & 0.10 & 0.01 \\
\hline \multirow[t]{2}{*}{4110} & 2.01 & 0.29 & 1145 & 0.03 & 0.00 & 2020 & 0.10 & 0.00 \\
\hline & & & 1135 & 0.01 & 0.00 & & & \\
\hline \multicolumn{3}{|c|}{ North Tributary 1 (NT1) } & 1130 & 0.04 & 0.00 & \multicolumn{3}{|c|}{ Tributaries to NT4 } \\
\hline 1585 & 0.00 & 0.00 & 1125 & 0.00 & 0.00 & 2160 & 0.01 & 0.01 \\
\hline 1582 & 0.03 & 0.00 & 1120 & 0.00 & 0.00 & 2155 & 0.04 & 0.01 \\
\hline 1579 & 0.02 & 0.00 & 1115 & 0.00 & 0.00 & 2150 & 0.00 & 0.00 \\
\hline 1550 & 0.09 & 0.01 & 1110 & 0.01 & 0.00 & 2145 & 0.00 & 0.00 \\
\hline 1540 & 0.10 & 0.00 & 1100 & 0.03 & 0.00 & 2140 & 0.01 & 0.00 \\
\hline 1515 & 0.13 & 0.01 & 1095 & 0.00 & 0.00 & 2130 & 0.01 & 0.00 \\
\hline \multirow[t]{2}{*}{1510} & 0.14 & 0.00 & 1090 & 0.00 & 0.00 & 2120 & 0.00 & 0.00 \\
\hline & & & 1065 & 0.00 & 0.00 & 2110 & 0.00 & 0.00 \\
\hline \multicolumn{3}{|c|}{ Tributaries to NT1 } & 1060 & 0.02 & 0.00 & 2100 & 0.02 & 0.00 \\
\hline 1577 & 0.00 & 0.00 & 1045 & 0.01 & 0.00 & 2095 & 0.00 & 0.00 \\
\hline 1576 & 0.00 & 0.00 & 1040 & 0.00 & 0.00 & 2090 & 0.00 & 0.00 \\
\hline 1575 & 0.00 & 0.00 & 1035 & 0.00 & 0.00 & 2085 & 0.00 & 0.00 \\
\hline 1573 & 0.00 & 0.00 & 1030 & 0.01 & 0.00 & 2083 & 0.00 & 0.00 \\
\hline 1570 & 0.00 & 0.00 & 1025 & 0.01 & 0.00 & 2080 & 0.00 & 0.00 \\
\hline 1565 & 0.01 & 0.00 & 1020 & 0.01 & 0.00 & 2070 & 0.00 & 0.00 \\
\hline
\end{tabular}

8 Gaining, Losing, and Dry Stream Reaches at Bear Creek Valley,

Oak Ridge, Tennessee, March and September 1994 
Table 1. Discharge data during high base flow, March 14 through March 19, 1994, and low base flow, September 9 through September 29, 1994 at Bear Creek Valley, Oak Ridge, Tennessee--continued

\begin{tabular}{ccc}
\hline \multirow{2}{*}{ Site } & \multicolumn{2}{c}{ Discharge } \\
\cline { 2 - 3 } number & HBF & LBF \\
\hline Tributaries to & NT4 & \\
2068 & 0.09 & 0.00 \\
2067 & 0.00 & 0.00 \\
2065 & 0.01 & 0.00 \\
2055 & 0.00 & 0.00 \\
2040 & 0.00 & 0.00 \\
2035 & 0.00 & 0.00 \\
2027 & 0.00 & 0.00 \\
2025 & 0.00. & 0.00
\end{tabular}

\begin{tabular}{ccc}
\hline Site & \multicolumn{2}{c}{ Discharge } \\
\cline { 2 - 3 } number & HBF & LBF \\
\hline North Tributary & 6 (NT6) & \\
3110 & 0.02 & 0.00 \\
3080 & 0.07 & 0.00 \\
3070 & 0.09 & 0.00 \\
3060 & 0.01 & 0.00 \\
3050 & 0.08 & 0.00
\end{tabular}

\begin{tabular}{ccc}
\hline Site & \multicolumn{2}{c}{ Discharge } \\
\cline { 2 - 3 } number & HBF & LBF \\
\hline North Tributary 8 (NT8) & \\
4480 & 0.04 & 0.01 \\
4475 & 0.00 & 0.02 \\
4430 & 0.08 & 0.01 \\
4405 & 0.09 & 0.01 \\
& & \\
Tributaries to NT8 & \\
4505 & 0.00 & 0.00 \\
4495 & 0.00 & 0.00 \\
4490 & 0.01 & 0.00 \\
4455 & 0.03 & 0.01 \\
4450 & 0.01 & 0.00 \\
4445 & 0.00 & 0.00 \\
4440 & 0.01 & 0.01 \\
4435 & 0.04 & 0.00 \\
4425 & 0.00 & 0.00 \\
4420 & 0.01 & 0.01 \\
4415 & 0.00 & 0.00 \\
4410 & 0.02 & 0.01
\end{tabular}

North Tributary (NT4A)

Tributaries to NTG

$\begin{array}{lll}3155 & 0.00 & 0.00 \\ 3150 & 0.01 & 0.00 \\ 3145 & 0.01 & 0.01 \\ 3140 & 0.00 & 0.00 \\ 3135 & 0.00 & 0.00 \\ 3130 & 0.00 & 0.00 \\ 3120 & 0.01 & 0.00 \\ 3115 & 0.00 & 0.00 \\ 3105 & 0.01 & 0.00 \\ 3100 & 0.01 & 0.00 \\ 3095 & 0.01 & 0.00 \\ 3090 & 0.01 & 0.00 \\ 3085 & 0.00 & 0.00 \\ 3078 & 0.00 & 0.00 \\ 3075 & 0.01 & 0.00 \\ 3065 & 0.01 & 0.00 \\ 3055 & 0.00 & 0.00\end{array}$

Tributaries to NT5

$\begin{array}{lll}3198 & 0.00 & 0.00 \\ 3202 & 0.00 & 0.00 \\ 3205 & 0.00 & 0.00 \\ 3210 & 0.03 & 0.00 \\ 3215 & 0.00 & 0.00 \\ 3225 & 0.00 & 0.00\end{array}$

Tributaries to NT5

$\begin{array}{lll}3265 & 0.00 & 0.00 \\ 3260 & 0.01 & 0.00 \\ 3255 & 0.00 & 0.00 \\ 3250 & 0.01 & 0.00 \\ 3245 & 0.01 & 0.00 \\ 32235 & 0.00 & 0.00 \\ 3230 & 0.00 & 0.00\end{array}$

$\begin{array}{ccc}\text { North Tributary } 6 \text { (NT6) } & \\ 3165 & 0.02 & 0.00 \\ 3160 & 0.02 & 0.01 \\ 3125 & 0.04 & 0.01\end{array}$

$\begin{array}{ccc}\text { North Tributary } 7 \text { (NT7) } & \\ 4620 & 0.02 & 0.00 \\ 4615 & 0.00 & 0.00 \\ 4610 & 0.02 & 0.00 \\ 4605 & 0.00 & 0.00 \\ 4600 & 0.00 & 0.00 \\ 4595 & 0.06 & 0.00 \\ 4590 & 0.00 & 0.00 \\ 4585 & 0.00 & 0.00 \\ 4580 & 0.07 & 0.00 \\ 4545 & 0.10 & 0.00 \\ 4540 & 0.07 & 0.00 \\ 4535 & 0.06 & 0.00\end{array}$

$\begin{array}{ccc}\text { South Tributary } 1 \text { (ST1) } & \\ 2180 & 0.00 & 0.00 \\ 2175 & 0.00 & 0.00 \\ 2170 & 0.00 & 0.00\end{array}$

South Tributary 2 (ST2)

$\begin{array}{lll}3305 & 0.00 & 0.00 \\ 3300 & 0.00 & 0.00 \\ 3290 & 0.01 & 0.00\end{array}$

Tributaries to ST2

$\begin{array}{lll}3295 & 0.00 \quad 0.00\end{array}$

South Tributary 3 (ST3)

\begin{tabular}{|c|c|c|}
\hline 3035 & 0.01 & 0.00 \\
\hline 3025 & 0.37 & 0.00 \\
\hline 3015 & 0.44 & 0.03 \\
\hline 3010 & 0.36 & 0.13 \\
\hline \multicolumn{3}{|c|}{ ibutaries to ST3 } \\
\hline 3030 & 0.00 & 0.00 \\
\hline 3020 & 0.00 & 0.00 \\
\hline \multicolumn{3}{|c|}{ outh Tributary 4 (ST4) } \\
\hline 4125 & 0.00 & 0.00 \\
\hline
\end{tabular}


Table 1. Discharge data during high base flow, March 14 through March 19, 1994, and low base

flow, September 9 through September 29, 1994 at Bear Creek Valley, Oak Ridge, Tennessee--continued

\begin{tabular}{ccc}
\hline Site & \multicolumn{2}{c}{ Discharge } \\
\cline { 2 - 3 } number & HBF & LBF \\
\hline South Tributary & 4 (ST4) & \\
4120 & 0.00 & 0.00
\end{tabular}

South Spring 1 (SS1)

$1502 \quad 0.07 \quad 0.00$

South Spring 2 (SS2)

$\begin{array}{lll}2335 & 0.22 & 0.02 \\ 2341 & 0.23 & 0.02\end{array}$

Tributaries to SS2

$2337 \quad 0.06 \quad 0.00$

$2339 \quad 0.03 \quad 0.00$

$\begin{array}{ccc}\text { South Spring 3 } & \text { (SS3) } \\ 2320 & 0.17 & 0.01 \\ 2330 & 0.12 & 0.00\end{array}$

Tributaries to SS3

$2325 \quad 0.05 \quad 0.00$

South Spring 4 (SS4)

$\begin{array}{lll}3175 & 0.13 & 0.00 \\ 3180 & 0.13 & 0.00\end{array}$

South Spring 5 (SS5)

$\begin{array}{lll}4115 & 0.44 & 0.10\end{array}$

South Spring 6 (SS6)

4105

0.01

0.00

10 Gaining, Losing, and Dry Stream Reaches at Bear Creek Valley, Oak Ridge, Tennessee, March and September 1994 
Table 2. Stream reach classification during high base flow at Bear Creek Valley, Oak Ridge,

Tennessee, March 14 through March 19, 1994

[All discharge measurements in cubic feet per second]

\begin{tabular}{|c|c|c|c|c|c|c|c|}
\hline \multicolumn{2}{|c|}{$\begin{array}{c}\text { Site } \\
\text { number }\end{array}$} & \multirow{2}{*}{$\begin{array}{c}\text { Stream reach } \\
\text { class- } \\
\text { ification }\end{array}$} & \multirow{2}{*}{$\begin{array}{l}\text { Change in } \\
\text { discharge }\end{array}$} & \multicolumn{2}{|c|}{$\begin{array}{c}\text { Site } \\
\text { number }\end{array}$} & \multirow{2}{*}{$\begin{array}{c}\text { Stream reach } \\
\text { class- } \\
\text { ification }\end{array}$} & \multirow{2}{*}{$\begin{array}{l}\text { Change in } \\
\text { discharge }\end{array}$} \\
\hline Upstream & Downstream & & & Upstream & Downstream & & \\
\hline \multicolumn{4}{|l|}{ Bear Creek } & \multicolumn{2}{|c|}{ North Tributary 1 (NT1) } & & \\
\hline 1600 & 1500 & losing flow & -0.14 & 1565 & -- & contributing site & - \\
\hline 1500 & 1495 & no change in flow & 0.00 & 1576 & -- & contributing site & - \\
\hline 1495 & 1485 & gaining flow & 0.12 & 1577 & -- & contributing site & -- \\
\hline 1485 & 1005 & losing flow & -0.44 & 1545 & - & contributing site & - \\
\hline 1005 & 2345 & losing flow & -0.13 & 1540 & - & contributing site & -- \\
\hline 2345 & 2315 & gaining flow & 0.25 & 1505 & -- & contributing site & - \\
\hline 2315 & 2190 & losing flow & -1.34 & & & & \\
\hline 2190 & 2185 & losing flow & -0.20 & \multicolumn{2}{|c|}{ North Tributary 2 (NT2) } & & \\
\hline 2185 & 2015 & losing flow & -0.16 & 1150 & 1148 & gaining flow & 0.01 \\
\hline \multirow[t]{2}{*}{2015} & 3310 & losing flow & -0.28 & 1148 & 1145 & gaining flow & 0.01 \\
\hline & & & & 1155 & 1140 & losing flow & -0.02 \\
\hline 3310 & 3285 & no change in flow & 0.00 & 1135 & 1130 & gaining flow & 0.03 \\
\hline 3285 & 3190 & losing flow & -0.26 & 1140 & 1105 & gaining flow & 0.08 \\
\hline 3190 & 3185 & gaining flow & 0.26 & & & & \\
\hline 3185 & 3175 & losing flow & $-0.37^{\star}$ & . 1115 & 1110 & gaining flow & 0.01 \\
\hline \multirow[t]{2}{*}{3175} & 3170 & gaining flow & 0.38 & 1125 & 1120 & dry & 0.00 \\
\hline & & & & 1105 & 1080 & losing flow & -0.22 \\
\hline 3170 & 3045 & no change in flow & 0.00 & 1100 & 1085 & gaining flow & 0.03 \\
\hline 3045 & 3040 & losing flow & -0.26 & 1095 & 1090 & dry & 0.00 \\
\hline 3040 & 3005 & no change in flow & 0.00 & & & & \\
\hline 3005 & 4630 & no change in flow & 0.00 & 1080 & 1075 & gaining flow & 0.15 \\
\hline \multirow[t]{2}{*}{4630} & 4530 & gaining flow & 0.38 & 1075 & 1050 & losing flow & -0.04 \\
\hline & & & & 1065 & 1060 & gaining flow & 0.02 \\
\hline 4530 & 4525 & losing flow & -0.13 & 1050 & 1015 & no change in flow & 0.00 \\
\hline 4525 & 4520 & gaining flow & 0.09 & 1040 & 1035 & dry & 0.00 \\
\hline 4520 & 4400 & gaining flow & 0.17 & & & & \\
\hline \multirow[t]{2}{*}{4400} & 4110 & gaining flow & 0.51 & 1045 & 1030 & no change in flow & 0.00 \\
\hline & & & & 1025 & 1020 & losing flow & -0.01 \\
\hline \multicolumn{2}{|c|}{ North Tributary 1 (NT1) } & & & 1015 & 1010 & no change in flow & 0.00 \\
\hline 1585 & 1582 & gaining flow & 0.03 & 1070 & - & contributing site & - \\
\hline 1582 & 1579 & losing flow & -0.01 & 1055 & - & contributing site & - \\
\hline 1579 & 1550 & gaining flow. & 0.03 & & & & \\
\hline 1575 & 1573 & dry & 0.00 & \multicolumn{2}{|c|}{ North Tributary 3 (NT3) } & & \\
\hline \multirow[t]{2}{*}{1573} & 1570 & dry & 0.00 & 2310 & 2295 & gaining flow & 0.04 \\
\hline & & & & 2295 & 2290 & no change in flow & 0.00 \\
\hline 1570 & 1555 & gaining flow & 0.03 & 2290 & 2285 & no change in flow & 0.00 \\
\hline 1550 & 1540 & no change in flow & 0.00 & 2285 & 2225 & losing flow & -0.05 \\
\hline 1530 & 1525 & gaining flow & 0.01 & 2225 & 2210 & gaining flow & 1.07 \\
\hline 1525 & 1520 & losing flow & -0.01 & & & & \\
\hline \multirow[t]{2}{*}{1520} & 1515 & gaining flow & 0.03 & 2210 & 2195 & no change in flow & 0.00 \\
\hline & & & & 2260 & 2245 & gaining flow & 0.02 \\
\hline 1515 & 1510 & no change in flow & 0.00 & 2245 & 2240 & losing flow & -0.01 \\
\hline 1560 & - & contributing site & - & 2240 & 2230 & no change in flow & 0.00 \\
\hline
\end{tabular}


Table 2. Stream reach classification during high base flow at Bear Creek Valley, Oak Ridge,

Tennessee, March 14 through March 19, 1994--continued

[All discharge measurements in cubic feet per second]

\begin{tabular}{|c|c|c|c|c|c|c|c|}
\hline \multirow{2}{*}{\multicolumn{2}{|c|}{$\begin{array}{c}\text { All aiscnarge measurements in cubr } \\
\text { Site } \\
\text { number }\end{array}$}} & \multirow{3}{*}{$\begin{array}{c}\text { Stream reach } \\
\text { class- } \\
\text { ification }\end{array}$} & \multirow{3}{*}{$\begin{array}{l}\text { Change in } \\
\text { discharge }\end{array}$} & & & & \\
\hline & & & & \multicolumn{2}{|c|}{$\begin{array}{c}\text { Site } \\
\text { number }\end{array}$} & \multirow{2}{*}{$\begin{array}{c}\text { Stream reach } \\
\text { class- } \\
\text { ification } \\
\end{array}$} & \multirow{2}{*}{$\begin{array}{l}\text { Change in } \\
\text { discharge }\end{array}$} \\
\hline Upstream & Downstream & & & Upstream & Downstream & & \\
\hline \multicolumn{4}{|c|}{ North Tributary 3 (NT3) } & \multicolumn{2}{|c|}{ North Tributary 5 (NT5) } & & \\
\hline 2280 & 2275 & no change in flow & 0.00 & 3200 & 3195 & losing flow & -0.02 \\
\hline 2270 & -- & contributing site & - & 3265 & 3260 & dry & 0.01 \\
\hline 2235 & -- & contributing site & -- & 3260 & 3255 & losing flow & -0.01 \\
\hline 2205 & - & contributing site & - & 3255 & 3250 & gaining flow & 0.01 \\
\hline 2200 & - & contributing site & - & 3235 & 3230 & dry & 0.00 \\
\hline \multicolumn{4}{|c|}{ North Tributary 4 (NT4) } & 3230 & 3225 & dry & 0.00 \\
\hline 2150 & 2145 & dry. & 0.00 & 3225 & 3210 & gaining flow & 0.03 \\
\hline 2145 & 2140 & gaining flow & 0.01 & 3202 & 3198 & dry & 0.00 \\
\hline 2160 & 2155 & gaining flow & 0.03 & 3245 & - & contributing site & -- \\
\hline 2155 & 2135 & gaining flow & 0.02 & & & & \\
\hline \multirow[t]{2}{*}{2135} & 2125 & losing flow & -0.04 & \multicolumn{2}{|c|}{ North Tributary 6 (NT6) } & & \\
\hline & & & & 3165 & 3160 & no change in flow & 0.00 \\
\hline 2125 & 2050 & losing flow & -0.07 & 3160 & 3155 & losing flow & -0.02 \\
\hline 2050 & 2030 & no change in flow & 0.00 & 3155 & 3140 & losing flow & -0.01 \\
\hline 2030 & 2020 & no change in flow & 0.00 & 3140 & 3145 & gaining flow & 0.01 \\
\hline 2110 & 2100 & gaining flow & 0.02 & 3145 & 3120 & losing flow & -0.04 \\
\hline \multirow[t]{2}{*}{2085} & 2083 & dry & 0.00 & & & & \\
\hline & & & & 3120 & 3115 & losing flow & -0.01 \\
\hline 2083 & 2070 & dry & 0.00 & 3115 & 3110 & gaining flow & 0.02 \\
\hline 2095 & 2090 & dry & 0.00 & 3110 & 3080 & gaining flow & 0.05 \\
\hline 2067 & 2068 & gaining flow & 0.09 & 3080 & 3070 & no change in flow & 0.00 \\
\hline 2040 & 2035 & dry & 0.00 & 3070 & 3050 & no change in flow & 0.00 \\
\hline \multirow[t]{2}{*}{2027} & 2025 & dry & 0.00 & & & & \\
\hline & & & & 3100 & 3095 & no change in flow & 0.00 \\
\hline 2080 & - & contributing site & - & 3105 & 3095 & losing flow & -0.01 \\
\hline 2130 & - & contributing site & - & 3135 & 3130 & dry & 0.00 \\
\hline 2120 & - & contributing site & - & 3130 & 3125 & gaining flow & 0.04 \\
\hline 2055 & - & contributing site & - & 3065 & 3060 & no change in flow & 0.00 \\
\hline \multirow[t]{2}{*}{2065} & - & contributing site & - & & & & \\
\hline & & & & 3150 & - & contributing site & - \\
\hline \multicolumn{2}{|c|}{ North Tributary 4A (NT4A) } & & & 3085 & - & contributing site & - \\
\hline 2010 & 2006 & dry & 0.00 & 3078 & - & contributing site & - \\
\hline \multirow[t]{2}{*}{2006} & 2005 & dry & 0.00 & 3075 & - & contributing site & -- \\
\hline & & & & 3055 & -- & contributing site & - \\
\hline \multicolumn{8}{|c|}{ North Tributary 5 (NT5) } \\
\hline 3280 & 3275 & no change in flow & 0.00 & \multicolumn{2}{|c|}{ North Tributary 7 (NT7) } & & \\
\hline 3275 & 3270 & no change in flow & 0.00 & 4620 & 4610 & no change in flow & 0.00 \\
\hline 3270 & 3240 & losing flow & -0.02 & 4610 & 4580 & no change in flow & 0.00 \\
\hline 3240 & 3205 & losing flow & -0.06 & 4580 & 4545 & gaining flow & 0.03 \\
\hline \multirow[t]{3}{*}{3205} & 3200 . & gaining flow & 0.12 & 4545 & 4540 & losing flow & -0.03 \\
\hline & - & & & 4540 & 4535 & no change in flow & 0.00 \\
\hline & & & & 4600 & 4595 & gaining flow & 0.06 \\
\hline
\end{tabular}

12 Gaining, Losing, and Dry Stream Reaches at Bear Creek Valley, Oak Ridge, Tennessee, March and September 1994 
Table 2. Stream reach classification during high base flow at Bear Creek Valley, Oak Ridge,

Tennessee, March 14 through March 19, 1994--continued

[All discharge measurements in cubic feet per second]

\begin{tabular}{|c|c|c|c|c|c|c|c|}
\hline \multicolumn{2}{|c|}{$\begin{array}{c}\text { Site } \\
\text { number }\end{array}$} & \multirow{2}{*}{$\begin{array}{c}\text { Stream reach } \\
\text { class- } \\
\text { iffication }\end{array}$} & \multirow{2}{*}{$\begin{array}{l}\text { Change in } \\
\text { discharge }\end{array}$} & \multicolumn{2}{|c|}{$\begin{array}{c}\text { Site } \\
\text { number }\end{array}$} & \multirow{2}{*}{$\begin{array}{c}\text { Stream reach } \\
\text { class- } \\
\text { ification }\end{array}$} & \multirow{2}{*}{$\begin{array}{l}\text { Change in } \\
\text { discharge }\end{array}$} \\
\hline Upstream & Downstream & & & Upstream & Downstream & & \\
\hline \multicolumn{4}{|c|}{ North Tributary 7 (NT7) } & \multicolumn{4}{|c|}{ South Spring 2 (SS2) } \\
\hline 4605 & -- & contributing site & -- & 2341 & 2335 & no change in flow & 0.00 \\
\hline 4585 & -- & contributing site & -- & 2339 & 2337 & gaining flow & 0.03 \\
\hline \multirow[t]{2}{*}{4590} & - & contributing site & -- & & & & \\
\hline & & & & \multicolumn{2}{|c|}{ South Spring 3 (SS3) } & & \\
\hline \multicolumn{2}{|c|}{ North Tributary 8 (NT8) } & & & 2330 & 2320 & no change in flow & 0.00 \\
\hline 4515 & 4510 & no change in fiow & 0.00 & 2335 & - & contributing site & -- \\
\hline 4510 & 4500 & gaining flow & 0.01 & & & & \\
\hline 4500 & 4485 & no change in flow & 0.00 & \multicolumn{2}{|c|}{ South Spring 4 (SS4) } & & \\
\hline 4485 & 4480 & no change in flow & 0.00 & 3180 & - & contributing site & -- \\
\hline \multirow[t]{2}{*}{4480} & 4435 & no change in flow & 0.00 & & & & \\
\hline & & & & \multicolumn{2}{|c|}{ South Spring 5 (SS5) } & & \\
\hline 4435 & 4430 & gaining flow & 0.04 & 4115 & - & contributing site & - \\
\hline 4430 & 4405 & no change in flow & 0.00 & & & & \\
\hline 4455 & 4440 & losing flow & -0.03 & \multicolumn{2}{|c|}{ South Spring 6 (SS6) } & & \\
\hline 4445 & 4450 & gaining flow & 0.01 & 4105 & - & contributing site & - \\
\hline 4120 & 4410 & gaining flow & 0.02 & & & & \\
\hline 4430 & 4505 & no change in flow & 0.00 & & & & \\
\hline 4490 & - & contributing site & - & & & & \\
\hline 4495 & - & contributing site & - & & & & \\
\hline 4475 & - & contributing site & - & & & & \\
\hline 4425 & - & contributing site & - & & & & \\
\hline 4415 & - & contributing site & - & & & & \\
\hline \multicolumn{4}{|c|}{ South Tributary 1 (ST1) } & & & & \\
\hline 2180 & 2175 & dry & 0.00 & & & & \\
\hline 2175 & 2170 & dry & 0.00 & & & & \\
\hline \multicolumn{4}{|c|}{ South Tributary 2 (ST2) } & & & & \\
\hline 3305 & 3300 & dry & 0.00 & & & & \\
\hline 3300 & 3290 & gaining flow & 0.01 & & & & \\
\hline 3295 & - & contributing site & - & & & & \\
\hline \multicolumn{4}{|c|}{ South Tributary 3 (ST3) } & & & & \\
\hline 3035 & 3025 & gaining flow & 0.36 & & & & \\
\hline 3025 & 3015 & gaining flow & 0.07 & & & & \\
\hline 3015 & 3010 & losing flow & -0.08 & & & & \\
\hline 3020 & - & contributing site & - & & & & \\
\hline \multicolumn{4}{|c|}{ South Tributary 4 (ST4) } & & & & \\
\hline 4120 & 4125 & dry & 0.00 & & & & \\
\hline \multicolumn{8}{|c|}{ South Spring 1 (SS1) } \\
\hline 1502 & - & contributing site & - & $\dot{.}$ & & & \\
\hline
\end{tabular}


Table 3. Stream reach classification during low base flow at Bear Creek Valley, Oak Ridge,

Tennessee, September 9 through September 29, 1994

[All flow measurements in cubic feet per second]

\begin{tabular}{|c|c|c|c|c|c|c|c|}
\hline \multicolumn{2}{|c|}{$\begin{array}{c}\text { Site } \\
\text { number }\end{array}$} & \multirow{2}{*}{$\begin{array}{c}\text { Stream reach } \\
\text { class- } \\
\text { ification } \\
\end{array}$} & \multirow{2}{*}{$\begin{array}{l}\text { Change in } \\
\text { discharge }\end{array}$} & \multicolumn{2}{|c|}{$\begin{array}{c}\text { Site } \\
\text { number }\end{array}$} & \multirow{2}{*}{$\begin{array}{c}\text { Stream reach } \\
\text { class- } \\
\text { ification } \\
\end{array}$} & \multirow{2}{*}{$\begin{array}{l}\text { Change in } \\
\text { discharge }\end{array}$} \\
\hline Upstream & Downstream & & & Upstream & Downstream & & \\
\hline \multicolumn{4}{|l|}{ Bear Creek } & \multicolumn{2}{|c|}{ North Tributary 1 (NT1) } & & \\
\hline 1600 & 1500 & losing flow & -0.01 & 1565 & - & contributing site & - \\
\hline 1500 & 1495 & gaining flow & 0.01 & 1576 & -- & contributing site & - \\
\hline 1495 & 1485 & gaining flow & 0.02 & 1577 & - & contributing site & - \\
\hline 1485 & 1005 & losing flow & -0.02 & 1545 & - & contributing site & - \\
\hline 1005 & 2345 & no change in flow & 0.00 & 1540 . & - & contributing site & - \\
\hline 2345 & 2315 & losing flow & -0.05 & 1505 & - & contributing site & - \\
\hline 2315 & 2190 & gaining flow & 0.04 & & & & \\
\hline 2190 & 2185 & losing flow & -0.04 & North Tributa & $y 2$ (NT2) & & \\
\hline 2185 & 2015 & dry & 0.00 & 1150 & 1148 & dry & 0.00 \\
\hline \multirow[t]{2}{*}{2015} & 3310 & dry & 0.00 & 1148 & 1145 & dry & 0.00 \\
\hline & & & & 1155 & 1140 & dry & 0.00 \\
\hline 3310 & 3285 & dry & 0.00 & 1135 & 1130 & dry & . $\quad 0.00$ \\
\hline 3285 & 3190 & dry & 0.00 & 1140 & 1105 & dry & 0.00 \\
\hline 3190 & 3185 & dry & 0.00 & & & & \\
\hline 3185 & 3175 & dry & 0.00 & 1115 & 1110 & dry & 0.00 \\
\hline \multirow[t]{2}{*}{3175} & 3170 & dry & 0.00 & 1125 & 1120 & dry & 0.00 \\
\hline & & & & 1105 & 1080 & dry & 0.00 \\
\hline 3170 & 3045 & dry & 0.00 & 1100 & 1085 & dry & 0.00 \\
\hline 3045 & 3040 & dry & 0.00 & 1095 & 1090 & dry & 0.00 \\
\hline 3040 & 3005 & losing flow & -0.06 & & & & \\
\hline 3005 & 4630 & no change in flow & 0.00 & 1080 & 1075 & dry & 0.00 \\
\hline \multirow[t]{2}{*}{4630} & 4530 & no change in flow & 0.01 & 1075 & 1050 & dry & 0.00 \\
\hline & & & & 1065 & 1060 & dry & 0.00 \\
\hline 4530 & 4525 & losing flow & -0.08 & 1050 & 1015 & dry & 0.00 \\
\hline 4525 & 4520 & dry & 0.00 & 1040 & 1035 & dry & 0.00 \\
\hline 4520 & 4400 & gaining flow & 0.03 & & & & \\
\hline \multirow[t]{2}{*}{4400} & 4110 & gaining flow & 0.15 & 1045 & 1030 & dry & 0.00 \\
\hline & & & & 1025 & 1020 & dry & 0.00 \\
\hline \multicolumn{2}{|c|}{ North Tributary 1 (NT1) } & & & 1015 & 1010 & dry & 0.00 \\
\hline 1585 & 1582 & dry & 0.00 & 1070 & - & contributing site & \\
\hline 1582 & 1579 & dry & 0.00 & 1055 & - & contributing site & - \\
\hline 1579 & 1550 & no change in flow & 0.00 & & & & \\
\hline 1575 & 1573 & dry & 0.00 & \multicolumn{2}{|c|}{ North Tributary 3 (NT3) } & & \\
\hline \multirow[t]{2}{*}{1573} & 1570 & dry & 0.00 & 2310 & 2295 & dry & 0.00 \\
\hline & & & & 2295 & 2290 & dry & 0.00 \\
\hline 1570 & 1555 & dry & 0.00 & 2290 & 2285 & dry & 0.00 \\
\hline 1550 & 1540 & losing flow & -0.01 & 2285 & 2225 & dry & 0.00 \\
\hline 1530 & 1525 & dry & 0.00 & 2225 & 2210 & dry & 0.00 \\
\hline 1525 & 1520 & dry & 0.00 & & & & \\
\hline \multirow[t]{2}{*}{1520} & 1515 & gaining flow & 0.01 & 2210 & 2195 & dry & 0.00 \\
\hline & & & & 2260 & 2245 & dry & 0.00 \\
\hline 1515 & 1510 & losing flow & -0.01 & 2245 & 2240 & dry & 0.00 \\
\hline 1560 & - & contributing site & - & 2240 & 2230 & dry & 0.00 \\
\hline
\end{tabular}

14 Gaining, Losing, and Dry Stream Reaches at Bear Creek Valley, Oak Pidge, Tennessee, March and September 1994 
Table 3. Stream reach classification during low base flow at Bear Creek Valley, Oak Ridge,

Tennessee, September 9 through September 29, 1994--continued

\begin{tabular}{|c|c|c|c|c|c|c|c|}
\hline \multicolumn{2}{|c|}{$\begin{array}{c}\text { Site } \\
\text { number }\end{array}$} & \multirow{2}{*}{$\begin{array}{c}\text { Stream reach } \\
\text { class- } \\
\text { ification }\end{array}$} & \multirow{2}{*}{$\begin{array}{l}\text { Change in } \\
\text { discharge }\end{array}$} & \multicolumn{2}{|c|}{$\begin{array}{c}\text { Site } \\
\text { number }\end{array}$} & \multirow{2}{*}{$\begin{array}{c}\text { Stream reach } \\
\text { class- } \\
\text { ification }\end{array}$} & \multirow{2}{*}{$\begin{array}{l}\text { Change in } \\
\text { discharge }\end{array}$} \\
\hline Upstream & Downstream & & & Upstream & Downstream & & \\
\hline \multicolumn{4}{|c|}{ North Tributary 3 (NT3) } & \multicolumn{2}{|c|}{ North Tributary 5 (NT5) } & & \\
\hline 2280 & 2275 & dry & 0.00 & 3260 & 3255 & dry & 0.00 \\
\hline 2270 & -- & contributing site & - & 3255 & 3250 & dry & 0.00 \\
\hline 2235 & - & contributing site & - & 3235 & 3230 & dry & 0.00 \\
\hline 2205 & - & contributing site & - & 3230 & 3225 & dry & 0.00 \\
\hline 2200 & - & contributing site & - & 3225 & 3210 & dry & 0.00 \\
\hline \multicolumn{2}{|c|}{ North Tributary 4 (NT4) } & & & 3202 & 3198 & dry & 0.00 \\
\hline 2150 & 2145 & dry & 0.00 & 3245 & -- & contributing site & - \\
\hline 2145 & 2140 & dry & 0.00 & & & & \\
\hline 2160 & 2155 & no change in flow & 0.00 & \multicolumn{2}{|c|}{ North Tributary 6 (NT6) } & & \\
\hline 2155 & 2135 & losing flow &.-0.01 & 3165 & 3160 & gaining flow & 0.01 \\
\hline \multirow[t]{2}{*}{2135} & 2125 & dry & 0.00 & 3160 & 3155 & losing flow & -0.01 \\
\hline & & & & 3155 & 3140 & dry & 0.00 \\
\hline 2125 & 2050 & dry & 0.00 & 3140 & 3145 & gaining flow & 0.01 \\
\hline 2050 & 2030 & gaining flow & 0.01 & 3145 & 3120 & losing flow & -0.02 \\
\hline 2030 & 2020 & losing flow & -0.01 & & & & \\
\hline 2110 & 2100 & dry & 0.00 & 3120 & 3115 & dry & 0.00 \\
\hline \multirow[t]{2}{*}{2085} & 2083 & dry & 0.00 & 3115 & 3110 & dry & 0.00 \\
\hline & & & & 3110 & 3080 & dry & 0.00 \\
\hline 2083 & 2070 & dry & 0.00 & 3080 & 3070 & dry & 0.00 \\
\hline 2095 & 2090 & dry & 0.00 & 3070 & 3050 & dry & 0.00 \\
\hline 2067 & 2068 & dry & 0.00 & & & & \\
\hline 2040 & 2035 & dry & 0.00 & 3100 & 3095 & dry & 0.00 \\
\hline \multirow[t]{2}{*}{2027} & 2025 & dry & 0.00 & 3105 & 3095 & dry & 0.00 \\
\hline & & & & 3135 & 3130 & dry & 0.00 \\
\hline 2080 & - & contributing site & - & 3130 & 3125 & gaining flow & 0.01 \\
\hline 2130 & - & contributing site & $\cdots$ & 3065 & 3060 & dry & 0.00 \\
\hline 2120 & - & contributing site & - & & & & \\
\hline 2055 & - & contributing site & - & 3150 & - & contributing site & - \\
\hline \multirow[t]{2}{*}{2065} & - & contributing site & - & 3085 & - & contributing site & -- \\
\hline & & & & 3078 & - & contributing site & - \\
\hline \multicolumn{2}{|c|}{ North Tributary 4A (NT4A) } & & & 3075 & - & contributing site & - \\
\hline 2010 & 2006 & dry & 0.00 & 3055 & - & contributing site & -- \\
\hline \multirow[t]{2}{*}{2006} & 2005 & dry & 0.00 & & & & \\
\hline & & & & \multicolumn{2}{|c|}{ North Tributary 7 (NT7) } & & \\
\hline \multicolumn{2}{|c|}{ North Tributary 5} & & & 4620 & 4610 & dry & 0.00 \\
\hline 3280 & 3275 & gaining flow & 0.01 & 4610 & 4580 & dry & 0.00 \\
\hline 3275 & 3270 & no change in flow & 0.00 & 4580 & 4545 & dry & 0.00 \\
\hline 3270 & 3240 & losing flow & -0.01 & 4545 & 4540 & dry & 0.00 \\
\hline 3240 & 3205 & dry & 0.00 & 4540 & 4535 & dry & 0.00 \\
\hline \multirow[t]{2}{*}{3205} & 3200 & dry & 0.00 & & & & \\
\hline & & & & 4600 & 4595 & dry & 0.00 \\
\hline 3200 & 3195 & dry & 0.00 & 4605 & - & contributing site & - \\
\hline 3265 & 3260 & dry & 0.00 & 4585 & - & contributing site & - \\
\hline
\end{tabular}


Table 3. Stream reach classification during low base flow at Bear Creek Valley, Oak Ridge, Tennessee, September 9 through September 29, 1994--continued

\begin{tabular}{|c|c|c|c|c|c|c|c|}
\hline \multicolumn{2}{|c|}{$\begin{array}{c}\text { Site } \\
\text { number }\end{array}$} & \multirow{2}{*}{$\begin{array}{l}\text { Stream reach } \\
\text { class- } \\
\text { ification }\end{array}$} & \multirow{2}{*}{$\begin{array}{l}\text { Change in } \\
\text { discharge }\end{array}$} & \multicolumn{2}{|c|}{$\begin{array}{c}\text { Site } \\
\text { number }\end{array}$} & \multirow{2}{*}{$\begin{array}{l}\text { Stream reach } \\
\text { class- } \\
\text { ification }\end{array}$} & \multirow{2}{*}{$\begin{array}{l}\text { Change in } \\
\text { discharge }\end{array}$} \\
\hline Upstream & Downstream & & & Upstream & Downstream & & \\
\hline \multicolumn{2}{|c|}{ North Tributary 7 (NT7) } & & & \multicolumn{2}{|c|}{ South Spring 2 (SS2) } & & \\
\hline \multirow[t]{2}{*}{4590} & - & contributing site & - & 2341 & 2335 & no change in flow & 0.00 \\
\hline & & & & 2339 & 2337 & dry & 0.00 \\
\hline \multicolumn{8}{|c|}{ North Tributary 8 (NTB) } \\
\hline 4515 & 4510 & dry & 0.00 & \multicolumn{2}{|c|}{ South Spring 3 (SS3) } & & \\
\hline 4510 & 4500 & dry & 0.00 & 2330 & 2320 & gaining flow & 0.01 \\
\hline 4500 & 4485 & gaining flow & 0.01 & 2335 & -- & contributing site & - \\
\hline 4485 & 4480 & no change in flow & 0.00 & & & & \\
\hline \multirow[t]{2}{*}{4480} & 4435 & losing flow & -0.02 & \multicolumn{2}{|c|}{ South Spring 4 (SS4) } & & \\
\hline & & & & 3180 & - & contributing site & - \\
\hline 4435 & 4430 & losing flow & -0.01 & & & & \\
\hline 4430 & 4405 & losing flow & -0.01 & \multicolumn{2}{|c|}{ South Spring 5 (SS5) } & & \\
\hline 4455 & 4440 & no change in flow & 0.00 & 4115 & - & contributing site & - \\
\hline 4445 & 4450 & dry & 0.00 & & & & \\
\hline \multirow{2}{*}{4420} & 4410 & gaining flow & 0.01 & \multicolumn{2}{|c|}{ South Spring 6 (SS6) } & & \\
\hline & & & & 4105 & - & contributing site & - \\
\hline 4430 & 4405 & losing flow & 0.01 & & & & \\
\hline 4490 & -- & contributing site & - & & & & \\
\hline 4495 & - & contributing site & - & & & & \\
\hline 4475 & - & contributing site & -- & & & & \\
\hline 4425 & -- & contributing site & - & & & & \\
\hline 4415 & - & contributing site & - & & & & \\
\hline \multicolumn{8}{|c|}{ South Tributary 1 (ST1) } \\
\hline 2180 & 2175 & dry & 0.00 & & & & \\
\hline 2175 & 2170 & dry & 0.00 & & & & \\
\hline \multicolumn{8}{|c|}{ South Tributary 2 (ST2) } \\
\hline 3305 & 3300 & dry & 0.00 & & & & \\
\hline 3300 & 3290 & dry & 0.00 & & & & \\
\hline 2175 & - & contributing site & - & & & & \\
\hline \multicolumn{8}{|c|}{ South Tributary 3 (ST3) } \\
\hline 3035 & 3025 & dry & 0.00 & & & & \\
\hline 3025 & 3015 & gaining flow & 0.03 & & & & , \\
\hline 3015 & 3010 & gaining flow & 0.10 & & & & \\
\hline 3020 & - & contributing site & - & & & & \\
\hline \multicolumn{8}{|c|}{ South Tributary 4 (ST4) } \\
\hline 4120 & 4125 & dry & 0.00 & & & & \\
\hline \multicolumn{8}{|c|}{ South Spring 1 (SS1) } \\
\hline 1502 & - & contributing site & - & & & & \\
\hline
\end{tabular}

16 Gaining, Losing, and Dry Stream Reaches at Bear Creek Valley, Oak Ridge, Tennessee, March and September 1994 


\section{SUMMARY}

In 1995 the U.S. Geological Survey, in cooperation with the U.S. Department of Energy, began a study to delineate stream reaches that were gaining flow, losing flow, or that were dry within a 1,125-acre study area which includes the uppermost part of Bear Creek and many tributaries north and south of Bear Creek. Discharge data from a seepage investigation at Bear Creek Valley, Oak Ridge, Tennessee, are presented in this report. The classification of stream reaches are based on discharge data at 229 sites along upper Bear Creek and its tributaries. These data were collected during high base flow, from March 14 through March 19, 1994, and during low base flow, from September 9 through September 29, 1994.

To identify reaches that gain or lose flow, the following criteria were used: for streamflow of less than 0.1 cubic foot per second $\left(\mathrm{ft}^{3} / \mathrm{s}\right)$, a change in flow of more than 25 percent of total flow was used to determine if the flow was increasing or decreasing; for streamflow equal to or greater than $0.1 \mathrm{ft}^{3} / \mathrm{s}$, a change in flow of 10 percent was used as the criterion. If two adjacent stream measurement sites had zero flow, these stream reaches were classified as dry.

During high base flow, discharge measurements for Bear Creek ranged from 0.01 to $2.01 \mathrm{ft}^{3} / \mathrm{s}$. Gaining and losing stream reaches occurred along Bear Creek in the range of 0.09 and $0.51 \mathrm{ft}^{3} / \mathrm{s}$ and 0.13 to $1.34 \mathrm{ft}^{3} / \mathrm{s}$, respectively. No dry stream reaches were observed along Bear Creek during high base flow. The discharge measurements along the north tributaries ranged from less than 0.01 to $1.16 \mathrm{ft}^{3} / \mathrm{s}$. All tributaries north of Bear Creek had gaining and losing stream reaches. Gains in flow for the north tributaries were in the range of 0.01 to $1.07 \mathrm{ft}^{3} / \mathrm{s}$, and losses in flow were in the range of 0.01 to $0.22 \mathrm{ft}^{3} / \mathrm{s}$. Discharge measurements along the south tributaries were mostly less than $0.25 \mathrm{ft}^{3} / \mathrm{s}$, with the exception of ST3 and ST5. Dry stream reaches were observed along ST1, ST2, and ST4. The south spring tributaries, SS1 through SS6, were mostly single sites with discharge measurements in the range of 0 to $0.44 \mathrm{ft}^{3} / \mathrm{s}$.

During low base flow, discharge measurements along Bear Creek ranged from 0.00 to $0.29 \mathrm{ft}^{3} / \mathrm{s}$. Streamflow gains and losses along Bear Creek were generally in the range of 0.01 to $0.10 \mathrm{ft}^{3} / \mathrm{s}$. The section of Bear Creek near NT4, NT5, and NT6 remained dry. Two stream reaches along Bear Creek near NT7 were losing flow $\left(0.06\right.$ and $\left.0.08 \mathrm{ft}^{3} / \mathrm{s}\right)$. One gaining stream reach along Bear Creek near NT8 had an increase of flow of $0.15 \mathrm{ft}^{3} / \mathrm{s}$. Only one site in the north tributaries had a discharge of $0.02 \mathrm{ft}^{3} / \mathrm{s}$. All other sites were dry or had a discharge of $0.01 \mathrm{ft}^{3} / \mathrm{s}$. North tributaries NT2, NT3, and NT7 were dry. South tributaries ST1, ST2, and ST4 remained dry during low base flow, and ST3 had discharge measurements of 0.03 and $0.13 \mathrm{ft}^{3} / \mathrm{s}$. South spring tributaries SS2, SS3, and SS5 had discharges of $0.02 \mathrm{ft}^{3} / \mathrm{s}, 0.01 \mathrm{ft}^{3} / \mathrm{s}$, and $0.10 \mathrm{ft}^{3} / \mathrm{s}$, respectively.

\section{REFERENCES CITED}

McMaster, W.M., 1967, Hydrologic data for the Oak Ridge area Tennessee: U.S. Geological Survey Water-Supply Paper 1839-N, $90 \mathrm{p}$.

Robinson, J.A., and Johnson, G.C., 1996, Results of a seepage investigation at Bear Creek Valley, Oak Ridge, Tennessee, January through September 1994: U.S. Geological Survey Open File Report 95-459, 45 p. 


\section{NOTICE}

Page(s) size did not permit electronic reproduction. Information may be purchased by the general public from the National Technical Information Service, U.S. Department of Commerce, Springfield, VA 22161 (Area Code 703-487-4650). DOE and DOE contractors may purchase information by contacting DOE's Office of Scientific and Technical Information, P.O. Box 62, Oak Ridge, TN 37831, Attn: Information Services (Area Code 423-576-8401). 